were compared to the 62 with successful testing. Infants were divided into two groups based on gestation, 38 weeks and below and 39 weeks and above. Results were dichotomised into sufficient and insufficient (QNS) results. The Chi square test indicated that there is a statistically significant association between gestational age and sufficient or insufficient result. $(\mathrm{p}=0.004)$

Discussion It would be helpful to compare our data to the same cohort in other centres and national rate to see if this is an acceptable QNS rate and obtain further data regarding gestational age. Collection techniques and laboratory analysis have already been optimised with current equipment and staff. Consideration could be given to delay offering sweat test by one to two weeks to those born at 37 and 38 weeks gestation in infants who only have 1 mutation. This may reduce need for time consuming and stressful repeat testing.

\section{GP277 EVALUATION OF A NOVEL DISPOSABLE OSCILLATING POSITIVE EXPIRATORY PRESSURE (OPEP) DEVICE IN A COHORT OF CHILDREN WITH CYSTIC FIBROSIS}

1,2J O'Sullivan Kevin*, 'Valerie Power, ${ }^{2,3}$ Deirdre McGrath, ${ }^{2}$ Colum Dunne, 'Leonard O'Sullivan, 3,4Barry Linnane. 'School of Design, University of Limerick, Limerick, Ireland; ${ }^{2}$ Graduate Entry Medical School, University of Limerick, Limerick, Ireland; ${ }^{3}$ University Hospital Limerick, Limerick, Ireland; ${ }^{4}$ National Childrens Research Centre, Dublin, Ireland

\subsection{6/archdischild-2019-epa.336}

Objectives To evaluate use of a novel disposable Oscillating Positive Expiratory Pressure (OPEP) device (SoloPep) by children with CF.

Methods A convenience sample of 36 participants, all current OPEP device users, was recruited from a paediatric CF service. For one month, participants replaced their current OPEP devices (typically used daily for up to 6 months) with the SoloPep device (daily disposable), and followed their existing OPEP regimen. Lung function was assessed on the day that use of the new device was adopted and following completion of the test period using the EasyOne Air Spirometer (NDD Medzintechnik AG, Switzerland). Lung Clearance Index (LCI) was assessed via multiple-breath nitrogen washout (Exhalyser D, Eco Medics, Switzerland). User experience of SoloPep was evaluated with a post-study questionnaire, rated on five-point Likert scales. All participants were trained at the first clinic visit to use the SoloPep device utilising a proprietary software program.

Results 31 participants completed the study: 13 females; median age 10 years, range 4-16 years. Lung function (mean $\pm \mathrm{SD} ; \% \mathrm{FEV} 1$, baseline $=83.26 \pm 19.63$, follow-up $=86.31$ $\pm 20.95 ; \% \mathrm{FVC}$, baseline $=92.65 \pm 16.02$, follow-up $=$ $94.32 \pm 16.82 ; \mathrm{FEV}_{1}: \mathrm{FVC}$, baseline $=0.79 \pm 0.11$, followup $=0.80 \pm 0.10)$ and LCI (mean \pm SD; baseline $=10.05$ \pm 2.86 , follow-up $=9.66 \pm 2.10)$ were unchanged post-intervention. Most participants felt that SoloPep was easy to use (72\% Strongly Agree; 24\% Agree; 4\% Neutral), addressed issues they had with cleaning their current OPEP device $(72 \%$ Strongly Agree; 16\% Agree; 12\% Neutral), and encouraged them to perform their OPEP therapy more regularly $(52 \%$ Strongly Agree; 28\% Agree; 16\% Neutral, 4\% Strongly Disagree). All participants agreed that they would like to use SoloPep as their usual OPEP device $(76 \%$ Strongly Agree; 24\% Agree). No adverse device-related events were reported during SoloPep use.
Conclusion SoloPep was developed as a daily disposable OPEP device to reduce the risk of infection due to poor device cleaning. It is a convenient, user-friendly alternative to currently available OPEP devices. Over the course of the study, lung function was maintained, no adverse events were reported, and positive user experiences of the device were reported. Therefore, SoloPep may be a useful adjunct to aid airway clearance in children with CF.

\section{GP278 WARD LEVEL HIGH FLOW NASAL CANNULA OXYGEN FOR THE MANAGEMENT OF BRONCHIOLITIS: THE IMPACT ON HIGH DEPENDENCY UNIT BEDS (INTERIM RESULTS)}

Natalie Bee*, Christopher Tee, Mairi Stark. Royal Hospital for Sick Children, Edinburgh, UK

10.1136/archdischild-2019-epa.337

Background Many units across the UK and Europe still use $\mathrm{HFNCO}_{2}$ only within the critical care department. Prior to the introduction of ward level high flow nasal cannula oxygen $\left(\mathrm{HFNCO}_{2}\right)$ ten percent of patients admitted with a diagnosis of bronchiolitis required high dependency (HDU) care in our tertiary centre. A review of winter 2016 data suggested that the introduction of ward $\mathrm{HFNCO}_{2}$ had the potential to reduce over a third of HDU admissions. The median length of stay in HDU was 7.82 days for bronchiolitis patients.

Aim To introduce ward level $\mathrm{HFNCO}_{2}$ for the management of bronchiolitis and audit the impact on critical care floor beds (HDU).

Methods

1. Produced an evidence-based guideline for $\mathrm{HFNCO}_{2}$ use at ward level in the management of bronchiolitis.

2. Set up a working group and instituted staff training.

3. Sought management approval leading to the funding of 2 machines for ward level use.

4. Implemented the protocol in September 2018.

5. Interim audit of the use and outcomes December 2018.

Results Twenty patients received ward $\mathrm{HFNCO}_{2}$. Only one patient had a significant background comorbidity which was neuromuscular. Table 1 shows the demographics, management and outcomes. Three patients required HDU care for continuous positive airway pressure. No patients required intensive care.

Overall therefore were 60 days of ward $\mathrm{HFNCO}_{2}$ use.

\begin{tabular}{ll} 
Abstract GP278 Table 1 & $\mathrm{N}=\mathbf{2 0}$ \\
\hline Demographics & $0.03-1.4$ \\
\hline Age range (decimal age) & 0.78 \\
Median decimal age (years) & 7 \\
Sex: Male & 13 \\
Female & 1 \\
Preterm & \\
Management & 1 \\
Oral feeds & 4 \\
NG continuous feeds & 15 \\
I.V fluids & \\
HFNCO ${ }_{2}$ & 3 \\
Mean no. of days of HFNCO2 & 3 \\
Deterioration requiring HDU ( $\mathrm{n}=$ ) & 0 \\
Deterioration requiring PICU ( $\mathrm{n}=$ ) & 5.9 \\
Mean length hospital stay (days) & 5 \\
Median length hospital stay (days) & \\
\hline
\end{tabular}


Conclusion The interim audit has shown that we have already made one HDU bed available for other patients for the equivalent of 2 months during the busiest period of the year when critical care beds are at crisis point. There were no adverse events during this new initiative and the median length of hospital stay for this patient group was not increased.

\section{GP279 A REVIEW OF THE DIAGNOSTIC EVALUATION OF COMPLICATED PARAPNEUMONIC EFFUSION OR EMPYEMA IN AN IRISH TERTIARY HOSPITAL}

'Oksana Kozdoba*, 'Patrick Gavin, ${ }^{2}$ Richard Drew, ${ }^{1}$ Des Cox. 'Our Ladys Children's Hospital Crumlin, Dublin, Ireland; ${ }^{2}$ Irish Meningitis ans Sepsis Reference Laboratory Temple Street Childrens University Hospital, Dublin, Ireland

\subsection{6/archdischild-2019-epa.338}

Introduction Complicated parapneumonic effusion or empyema is a relatively common complication of pneumonia, often requiring thoracocentesis. The diagnostic yield with traditional culture of blood or pleural aspirate specimens is low, emphesizing the role for new molecular techniques to improve identification of the responsible pathogens.

Aim The purpose of this study was to review the laboratory investigation of childhood complicated parapneumonic effusion or empyema with the view to optimising diagnosis.

Methods A retrospective review of paediatric cases of complicated parapneumonic effusion or empyema requiring thoracocentesis was undertaken in an acute tertiary referral paediatric hospital, over a five year period, from January 2014 to December 2018. Cases with clinical and radiographic findings consistent with a diagnosis of complicated parapneumonic effusion or empyema were only included if a sterile site specimen was taken for diagnostic microbiologic evaluation. Baseline patient demographic data, clinical findings, laboratory indices, microbiology results and imaging findings were collected.

Results Sterile site specimens from 43 children with parapneumonic effusion/empyema were identified (females,60\%). $79 \%$ of the children were younger then 5 years of age. $45 \%$ (14 of 31 ) of children who had virologic testing performed had at least one respiratory virus detected. Six children had multiple viruses detected. A causative bacteria was identified in 24 cases $(56 \%), 6$ by conventional culture (pleural fluid, 5; blood,1) and 21 by PCR (pleural fluid, 20; blood, 3). Three children had both culture and PCR positive. PCR had the highest detection rate of causative organism: pleural fluid PCR positive, $52 \%$ (20 of 38 tested); blood PCR positive, 50\% (3 of 6 tested). Pleural fluid culture positive, $11.6 \%$ (5 of 43 tested); and blood culture positive $2.5 \%$ (1 of 39 tested). Streptococcus pneumonia was the causative organism detected in $95 \%$ cases.

Conclusion This retrospective review confirms that in paediatric cases of complicated parapneumonic effusion or empyema traditional microbiological culture of sterile site specimens infrequently identifies a causative organism. For such culture negative cases, appropriate PCR testing significantly improves the detection rate of causative organisms.

\section{GP280 SLEEP DISORDERED BREATHING AND SPINA BIFIDA - EXPERIENCE IN A TERTIARY REFERRAL PAEDIATRIC CENTRE}

Aoiibhinn Walsh*, Angela Mernagh, John Caird, Darach Crimmins, Fiona Ringholz, Michael Williamson, Fiona Healy. Temple Street Children's University Hospital, Dublin, Ireland

10.1136/archdischild-2019-epa.339

Introduction There is a paucity of knowledge regarding Sleep Disordered Breathing (SDB) in paediatric patients with Spina Bifida/Myelomeningocoele (SB/MMC). Undiagnosed, untreated SDB has detrimental effects on growth, development (1) and cognition (2) which is especially important to recognise in those with other co-morbidities as is the case in $\mathrm{SB} / \mathrm{MMC}$.

Aim There are currently 317 patients aged 0-16 years attending the Spina Bifida service at Temple Street Children's University (TSCUH). Fourteen of 317 patients (4.4\%) currently require Non-Invasive Ventilation (NIV) therapy for SDB under the care of the Respiratory Team. We hypothesize that a subpopulation of the SB/MMC patients are at risk of undiagnosed SDB. We aimed to initially review the demographics of the sub-group of SB/MMC patients currently requiring NIV by assessing for potential predictors of SDB.

Method We used up to date medical records of the patients under our care to look for potential predictors for SDB, in particular hydrocephalus with VP shunt, Chiari II malformation and intellectual disability (ID). We then compared our data to what is known about the general SB/MMC population.

Results $100 \%$ of SB patients with SDB requiring NIV had hydrocephalus with VP shunt compared to $80 \%$ in general SB/MMC patients. 92\% also had Chiari II malformation. 50\% had a moderate to severe ID in contrast to $25-25 \%$ of the general SB/MMC population.

Conclusion A higher proportion of SB/MMC patients known to have SDB have hydrocephalus and ID compared to the SB/ MMC population mean. At present there is no standardised screen for SDB in our SB/MMC patients. The results of this review have prompted a clinical audit of all of SB/MMC patients at TSCUH to identify those at higher risk of SDB and ultimately determine the prevalence of SDB in this population.

\section{REFERENCES}

1. 'How do we recognize the child with OSAS?' Joosten KF, et al. Pediatric Pulmonology 2017 Feb;52(2):260-271.

2. 'Association of Sleep-Disordered Breathing With Cognitive Function and Risk of Cognitive Impairment: A Systematic Review and Meta-analysis.' Leng Y, et al. JAMA Neurology 2017 Oct 1;74(10):1237-1245. 